\title{
Fascitis necrotizante como complicación secundaria a dehiscencia anastomótica tardía tras resección anterior de recto
}

\author{
Necrotizing fasciitis as a complication of late anastomotic leak after anterior resection for \\ rectal cancer \\ Víctor Domínguez-Prieto ${ }^{1 *}$, Miguel León-Arellano ${ }^{1}$ Alicia Alvarellos-Pérez ${ }^{1}$, Mario Ortega-López ${ }^{1}$ y \\ Carlos Pastor ${ }^{2}$ \\ ${ }^{1}$ Unidad de Cirugía Colorrectal, H.U. Fundación Jiménez Díaz; ${ }^{2}$ Unidad de Cirugía Colorrectal, Clínica Universidad de Navarra. Madrid, España
}

\section{Resumen}

Antecedentes: La dehiscencia anastomótica es una complicación grave de la cirugía del cáncer de recto que conlleva un aumento de la morbimortalidad. Su incidencia se sitúa en un 3-21\%, manifestándose habitualmente alrededor del quinto a séptimo días de posoperatorio, si bien existen casos de presentación tardía en forma de fístulas o sinus anastomóticos crónicos. Casos clínicos: Presentamos tres casos de pacientes intervenidos mediante resección anterior por cáncer de recto que desarrollaron fascitis necrotizante secundaria a dehiscencia anastomótica tardía. Conclusiones: Creemos recomendable el tratamiento quirúrgico resolutivo y precoz de las fístulas y sinus anastomóticos crónicos, incluso asintomáticos, por el potencial riesgo de fascitis necrotizante que suponen.

Palabras clave: Cáncer de recto. Dehiscencia anastomótica. Fuga anastomótica. Fascitis necrotizante.

\begin{abstract}
Background: Anastomotic leak is a serious complication of rectal cancer surgery that leads to increased morbidity and mortality. Its incidence is 3-21\%, usually appearing 5-7 days after surgery, although there are cases of late presentation as chronic anastomotic fistulas or sinuses. Case report: We present three cases of patients who underwent anterior resection for rectal cancer and developed necrotizing fasciitis due to late anastomotic leaks. Conclusions: We believe that early and resolutive surgical treatment is recommended for chronic anastomotic fistulas or sinuses, even when asymptomatic, because of the associated risk of necrotizing fasciitis.
\end{abstract}

Key words: Rectal cancer. Anastomotic leak. Anastomotic leakage. Necrotizing fascitis. 


\section{Introducción}

La dehiscencia anastomótica (DA) es una complicación grave de la cirugía del cáncer de recto $(\mathrm{CR})$ que conlleva un aumento de la morbimortalidad posoperatoria (mortalidad de hasta el $30 \%$ ) y de la estancia hospitalaria ${ }^{1-4}$, así como un retraso en el inicio del tratamiento oncológico adyuvante que puede influir en la supervivencia.

Su incidencia en la resección anterior de recto (RAR) se sitúa entre el $3 \%$ y el $21 \%^{4}$, aunque es variable en la literatura, sin que se hayan descrito diferencias significativas entre anastomosis manuales y mecánicas o con el empleo de estomas derivativos ${ }^{5}$, si bien estos últimos minimizan sus consecuencias.

Existen factores que predicen un mayor riesgo de DA, como la altura del tumor y la distancia de la anastomosis al margen anal, la radioterapia preoperatoria, los estadios localmente avanzados, la edad, el sexo masculino, la obesidad, el tabaquismo y la desnutrición, entre otros ${ }^{1-6}$.

La mayoría de los casos se presentan a los 5-7 días de posoperatorio en forma de íleo paralítico, fiebre, taquicardia, signos de peritonitis y sepsis, requiriendo con frecuencia tratamiento quirúrgico urgente. Adicionalmente, se han descrito casos de presentación tardía, incluso meses o años tras la cirugía ${ }^{7-9}$, de manera que hasta un $12 \%$ ocurren después del día 30 de posoperatorio ${ }^{8}$ en forma de fístulas o sinus anastomóticos crónicos prácticamente asintomáticos.

\section{Casos clínicos}

Se presentan tres pacientes con $\mathrm{CR}$ intervenidos mediante RAR laparoscópica que desarrollaron DA tardía en forma de fístulas y sinus anastomóticos, y secundariamente cuadros de fascitis necrotizante (FN) por extensión del foco infeccioso pélvico hacia las extremidades inferiores.

\section{Paciente 1}

Varón de 76 años, intervenido mediante RAR, con anastomosis colorrectal mecánica y colostomía lateral transversa de protección por CR medio T3N2 tras quimiorradioterapia neoadyuvante. Fue dado de alta el día 11 de postoperatorio.

El día 23 de postoperatorio acudió a urgencias por fiebre y dolor en el muslo izquierdo. Se realizó una tomografía computarizada (TC) que mostró DA y la

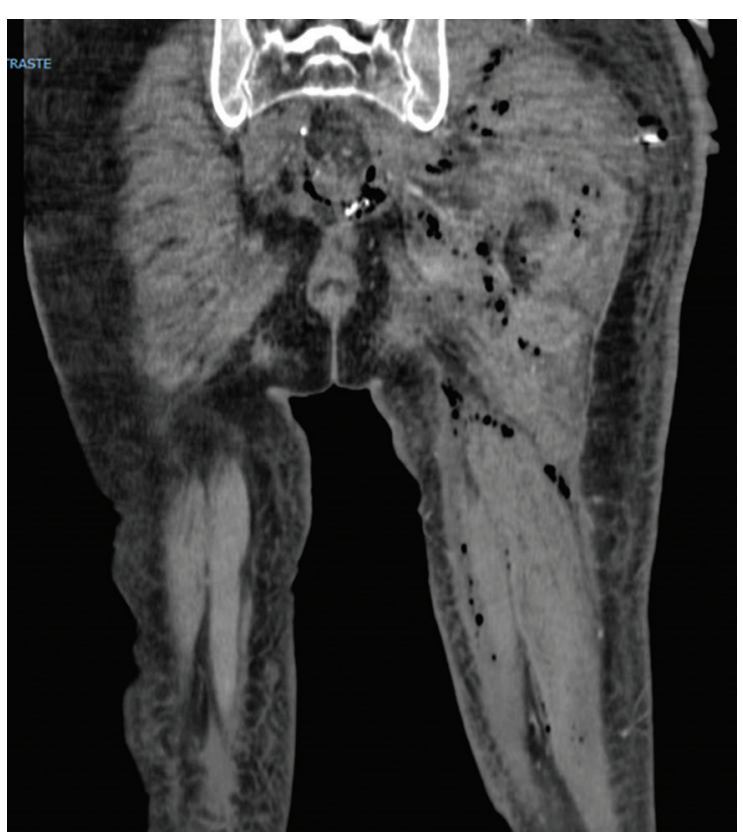

Figura 1. Dehiscencia de la anastomosis colorrectal y presencia de gas en raíz de miembro inferior izquierdo (paciente 1).

presencia de un trayecto fistuloso en la cara posterior de la anastomosis, así como gas extraluminal y signos inflamatorios con extensión a través de la escotadura ciática izquierda a la región glútea y el plano muscular del muslo izquierdo, en relación con FN (Fig. 1).

Evolucionó favorablemente tras 13 días de ingreso en la unidad de cuidados intensivos (UCl), antibioticoterapia de amplio espectro, colostomía terminal y varios desbridamientos quirúrgicos, hasta el alta hospitalaria a los 62 días de ingreso.

\section{Paciente 2}

Mujer de 69 años, intervenida mediante RAR transesfinteriana, con anastomosis coloanal manual e ileostomía de protección por CR bajo T3N1b, sin quimiorradioterapia neoadyuvante previa. Fue dada de alta el día 5 de posoperatorio sin incidencias.

El día 14 de postoperatorio acudió a urgencias por malestar general, dolor anal y fiebre. Se realizó una TC que mostró la presencia de una DA con una colección presacra que fue manejada de forma conservadora mediante antibioticoterapia y drenaje por vía transanal, con buena evolución posterior hasta el alta.

Tras recibir quimioterapia adyuvante, reconstrucción del tránsito intestinal y 18 meses de seguimiento en consulta, acudió nuevamente a urgencias por malestar general, fiebre y dolor glúteo izquierdo. 


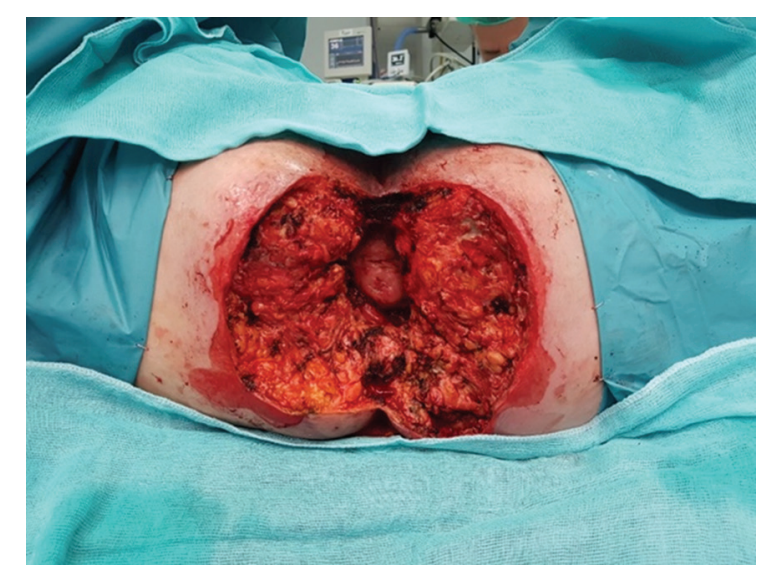

Figura 2. Amputación abdominoperineal y desbridamiento quirúrgico (paciente 2).

Se realizó una nueva TC en la que se observaron varios trayectos fistulosos perirrectales con extensión hacia la región glútea izquierda, burbujas aéreas y signos inflamatorios acompañantes, en relación con gangrena de Fournier.

Evolucionó favorablemente tras 6 días de ingreso en la $\mathrm{UCl}$, antibioticoterapia de amplio espectro, amputación abdominoperineal (Fig. 2) y varios desbridamientos quirúrgicos, hasta el alta hospitalaria a los 17 días de ingreso.

\section{Paciente 3}

Mujer de 68 años, intervenida mediante RAR, con anastomosis coloanal manual e ileostomía de protección por CR bajo T2N1 tras quimiorradioterapia neoadyuvante. Tras quimioterapia adyuvante y reconstrucción del tránsito a los 8 meses de la cirugía, desarrolló una fístula rectovaginal oligosintomática que fue manejada de forma conservadora mediante seguimiento anual en consulta.

A los 5 años y 7 meses de la cirugía inicial acudió a urgencias por dolor y aumento de volumen en el muslo derecho.

Se realizó una TC que mostró la presencia de un trayecto fistuloso anastomótico con cambios inflamatorios y burbujas aéreas en la musculatura glútea, el psoas ilíaco y la musculatura de muslo derecho, hallazgos en relación con FN secundaria a la fístula anastomótica.

A pesar del tratamiento intensivo mediante antibioticoterapia de amplio espectro, amputación abdominoperineal y varios desbridamientos quirúrgicos, la paciente falleció a los 26 días de ingreso en la $\mathrm{UCl}$.

En los cultivos de las heridas se obtuvo crecimiento de flora mixta intestinal y Enterococcus faecium (paciente 1), Prevotella spp. (paciente 2) y Escherichia coli y Streptococcus viridans (paciente 3).

\section{Discusión}

La DA es una complicación grave de la cirugía del CR que requiere con frecuencia un manejo quirúrgico precoz debido a su alta morbimortalidad (mortalidad de hasta el $30 \%)^{1-4}$. Si bien su presentación clínica tiene lugar habitualmente durante los primeros días tras la cirugía, en forma de peritonitis y sepsis, se han descrito casos de presentación tardía (hasta un $12 \%$ después de 30 días de posoperatorio) ${ }^{8}$, incluso meses o años tras la intervención ${ }^{7-9}$, en forma de fístulas y sinus anastomóticos crónicos que suelen cursar de forma oligosintomática.

Numerosos factores se han asociado con un mayor riesgo de DA, entre los que destaca la localización de la anastomosis: el riesgo es mayor en anastomosis más distales, más cercanas al margen anal, que en aquellas más proximales ${ }^{1-3,5,6}$. La presentación clínica dependerá de la localización intraperitoneal o extraperitoneal de la anastomosis, de modo que en las DA intraperitoneales predominan los signos de peritonitis y sepsis, mientras que las de localización extraperitoneal, como frecuentemente ocurre en la RAR, cursan de un modo más larvado ${ }^{7}$, con frecuencia en forma de fístulas y sinus anastomóticos crónicos, en el $5 \%$ de las RAR a pesar del uso de estomas derivativos ${ }^{10}$. De este modo, aquellos casos de DA intraperitoneal que cursen en forma de peritonitis y sepsis requerirán un tratamiento quirúrgico agresivo y urgente, mientras que en casos de DA extraperitoneal, con presentación clínica más larvada, el manejo conservador mediante antibiótico o drenaje percutáneo, o ambos, es una opción de tratamiento aceptada. No obstante, el tratamiento de la DA comienza con su prevención mediante la optimización prequirúrgica de los pacientes y la corrección de los factores de riesgo modificables (cese del hábito tabáquico, pérdida de peso, optimización nutricional, etc.), así como una adecuada técnica quirúrgica (anastomosis sin tensión y con adecuada vascularización, comprobación de la estanqueidad mediante air test, protección mediante estomas proximales, etc. $)^{7}$.

No existe a día de hoy una definición exacta y consensuada para la DA tardía. Algunos autores consideran DA tardía aquella que ocurre tras el alta hospitalaria, mientras que otros reservan este término para la que ocurre pasados los primeros 210 incluso 30 días tras la cirugía. Su incidencia ha sido estimada en un $0.3-4.3 \%$, y supone una tercera parte de los casos totales de DA 9 . 
La FN y la gangrena de Fournier son infecciones graves rápidamente progresivas que afectan a la piel, los tejidos blandos y los planos musculofasciales, caracterizadas por la destrucción y la necrosis de los tejidos afectos, así como por una grave toxicidad sistémica, que conllevan una elevada morbimortalidad. Los microorganismos más frecuentemente implicados en su etiopatogenia son Staphylococcus spp. (Staphylococcus aureus), Streptococcus spp. (Streptococcus pyogenes), Bacteroides y E. coli, con frecuencia en forma de infecciones polimicrobianas ${ }^{11}$. Entre sus factores de riesgo destacan la diabetes, las enfermedades crónicas, la inmunosupresión, la edad mayor de 60 años, las enfermedades oncológicas y la obesidad $^{12}$.

La FN se ha descrito como complicación secundaria a DA tras una RAR por $\mathrm{CR}^{7,13}$, incluso años tras la cirugía en casos de DA de presentación tardía 7 . Se trata de una complicación muy infrecuente y apenas descrita en la literatura, aunque potencialmente muy grave, en la que el foco infeccioso pélvico progresa hacia las extremidades inferiores a través del orificio obturador o del orificio ciático, y que asocia una alta morbimortalidad (mortalidad sin tratamiento cercana al $100 \%$; con tratamiento, $15-52 \%)^{12}$. Por lo tanto, un diagnóstico precoz basado en un alto grado de sospecha clínica y un tratamiento quirúrgico agresivo mediante desbridamiento de los tejidos afectos, así como la antibioticoterapia de amplio espectro, resul$\tan$ fundamentales en su manejo $0^{711,12}$.

Presentamos tres casos de FN secundaria a DA tardía tras una RAR por CR, complicación apenas descrita en la literatura en este contexto, en los que la ausencia de un tratamiento quirúrgico agresivo de la DA tardía terminó condicionando el desarrollo de FN. La DA puede ocurrir en cualquier momento tras una RAR, incluso pasados meses o años en forma de DA tardía, por lo que, ante un paciente con antecedente de cirugía colorrectal, el clínico debe tener siempre en mente esta posibilidad. La persistencia de un foco infeccioso pélvico en forma de fístula o sinus anastomótico crónico secundarios a DA tardía supone una situación de riesgo, ya que puede condicionar la extensión de la infección a las extremidades inferiores a través del orificio obturador o del orificio ciático y el desarrollo de FN, complicación con una elevada morbimortalidad. Por ello, en pacientes con DA tardía y fístulas o sinus anastomóticos crónicos tras una RAR, creemos recomendado su tratamiento quirúrgico resolutivo y precoz incluso en casos asintomáticos o paucisintomáticos para evitar el desarrollo de FN y sus graves consecuencias.

\section{Responsabilidades éticas}

Protección de personas y animales. Los autores declaran que para esta investigación no se han realizado experimentos en seres humanos ni en animales.

Confidencialidad de los datos. Los autores declaran que han seguido los protocolos de su centro de trabajo sobre la publicación de datos de pacientes.

Derecho a la privacidad y consentimiento informado. Los autores han obtenido el consentimiento informado de los pacientes y/o sujetos referidos en el artículo. Este documento obra en poder del autor de correspondencia.

\section{Financiamiento}

El presente trabajo no ha recibido ningún tipo de financiamiento.

\section{Conflicto de intereses}

Los autores declaran no tener conflicto de intereses.

\section{Bibliografía}

1. Rao V, Ashok P, Sanjay M, Srinivas Rao S. Incidence, consequences, and risk factors for anastomotic dehiscence after colorectal surgery: a prospective monocentric study. JEBMH. 2018;5:1898-901.

2. Choi H, Law W, Ho J. Leakage after resection and intraperitoneal anastomosis for colorectal malignancy: analysis of risk factors. Dis Colon Rectum. 2006;49:1719-25.

3. Dietz D. Postoperative complications. En: Beck D, Roberts $P$ Saclarides T, Senagore A, Stamos M, editores. The ASCRS textbook of colon and rectal surgery. New York: Springer; 2011. p. 157-73.

4. Kanellos I, Vasiliadis K, Angelopoulos S, Tsachalis T, Pramateftakis M, Mantzoros I, et al. Anastomotic leakage following anterior resection for rectal cancer. Tech Coloproctol. 2004;8(Suppl 1):s79-s81.

5. Matthiessen P, Hallbook O, Andersson M, Rutegard J, Sjodahl R. Risk factors for anastomotic leakage after anterior resection of the rectum. Colorectal Dis. 2004;6:462-9.

6. Kingham T, Pachter H. Colonic anastomotic leak: risk factors, diagnosis, and treatment. J Am Coll Surg. 2009;208:269-78.

7. Nagib A, Kiffin C, Carrillo E, Rosenthal A, Solomon R, Davare D. Necrotizing fasciitis resulting from an anastomotic leak after colorectal resection. Case Rep Surg. 2018;2018:1-3.

8. Hyman N, Manchester T, Osler T, Burns B, Cataldo P. Anastomotic leaks after intestinal anastomosis. Ann Surg. 2007;245:254-8.

9. Iwamoto M, Kawada K, Hida K, Hasegawa S, Sakai Y. Delayed anastomotic leakage following laparoscopic intersphincteric resection for lower rectal cancer: report of four cases and literature review. World J Surg Onc. 2017;15:143.

10. Arumainayagam N, Chadwick M, Roe A. The fate of anastomotic sinuses after total mesorectal excision for rectal cancer. Colorectal Dis. 2009;11:288-90.

11. Goh $\mathrm{T}$, Goh LG, Ang $\mathrm{CH}$, Wong $\mathrm{CH}$. Early diagnosis of necrotizing fasciitis. Br J Surg. 2014;101:e119-25.

12. Yiasemidou M, Majumder S, Basheer M. Necrotising fasciitis after laparoscopic rectal cancer surgery. Ann R Coll Surg Engl. 2017;99:e123-4.

13. Řezáč $T$, Stašek M, Zbořil $P$, Vomáčková K, Bébarová L, Hanuliak J, et al. Necrotizing pelvic infection after rectal resection. A rare indication of endoscopic vacuum-assisted closure therapy. A case report. Int J Surg Case Rep. 2019;61:44-7. 